\title{
On the spectral properties of a Sturm-Liouville problem with eigenparameter in the boundary condition
}

\author{
Emir A. Maris ${ }^{1}$ (D), Sertac Goktas ${ }^{* 2}$ (1) \\ ${ }^{1}$ Vocational School of Technical Sciences, Mersin University, 33343, Mersin, Turkey \\ ${ }^{2}$ Department of Mathematics, Mersin University, 33343, Mersin, Turkey
}

\begin{abstract}
The spectral problem

$$
\begin{gathered}
-y^{\prime \prime}+q(x) y=\lambda y, \quad 0<x<1, \\
y(0)=0, \quad y^{\prime}(0)=\lambda\left(a y(1)+b y^{\prime}(1)\right),
\end{gathered}
$$

is considered, where $\lambda$ is a spectral parameter, $q(x) \in L_{1}(0,1)$ is a complex-valued function, $a$ and $b$ are arbitrary complex numbers which satisfy the condition $|a|+|b| \neq 0$. We study the spectral properties (existence of eigenvalues, asymptotic formulae for eigenvalues and eigenfunctions, minimality and basicity of the system of eigenfunctions in $\left.L_{p}(0,1)\right)$ of the above-mentioned Sturm-Liouville problem.
\end{abstract}

Mathematics Subject Classification (2010). 34B05, 34B24, 34L10, 34L20

Keywords. eigenvalues, eigenfunctions, minimal system, basis

\section{Introduction}

In this study, we shall consider the Sturm-Liouville problem

$$
\begin{gathered}
-y^{\prime \prime}+q(x) y=\lambda y, \quad 0<x<1, \\
y(0)=0, \\
y^{\prime}(0)=\lambda\left(a y(1)+b y^{\prime}(1)\right),
\end{gathered}
$$

where $\lambda$ is a spectral parameter, $q(x) \in L_{1}(0,1)$ is a complex-valued function, $a$ and $b$ are arbitrary complex numbers which satisfy the condition $|a|+|b| \neq 0$.

Researching various properties (existence of eigenvalues, asymptotic formulae for eigenvalues and eigenfunctions, minimality and basicity) of Sturm-Liouville operators is of great importance in the spectral theory of differential operators. Especially, the uniform convergence of Fourier series expansions of this operators can be also investigated with the help of the conclusions obtained by examining these properties in the next studies. So that, this investigation underlies an important class of the mathematical physics problems in applications. Therefore, firstly it is intended to investigate properties like the existence of eigenvalues, the asymptotic formulae of eigenvalues and eigenfunctions, and

\footnotetext{
*Corresponding Author.

Email addresses: e.ali.maris@gmail.com (E.A. Maris), srtcgoktas@gmail.com (S. Goktas)

Received: 06.11.2018; Accepted: 02.10.2019
} 
the basis properties of the system of eigenfunctions of the problem (1.1)-(1.3) in $L_{p}(0,1)$ $(1<p<\infty)$ in this article.

Sturm-Liouville problems with the boundary conditions depending on the spectral parameter were studied in order to investigate their various properties in many articles (see $[1,2,6-8,10-17,19-23,25])$. The problems on the basis property of system of root functions corresponding to Sturm- Liouville problems for some differential operators which contain different forms (linearly, rationally, quadratically etc.) of spectral parameter in one of the boundary conditions were considered in $[1,2,8,10,12,15,16,22]$.

The basis property of the system of eigenfunctions corresponding to the spectral problem

$$
\begin{gathered}
y^{\prime \prime}+\lambda y=0,0<x<1 \\
y(0)=0, y^{\prime}(0)-a \lambda y(1)=0 \quad(a>0)
\end{gathered}
$$

in the space $L_{p}(0,1)$ was proved in [22]. Then the basis property for the system of eigenfunctions of the boundary value problem

$$
\begin{gathered}
-y^{\prime \prime}+q(x) y=\lambda y, 0<x<1, \\
y(0)=0, y^{\prime}(0)-a \lambda y(1)=0 \quad(a \neq 0)
\end{gathered}
$$

in $L_{p}(0,1)$ was studied in [12]. It was also verified that the system of root functions of the problem (1.5) with one function deleted, is a basis in the space $L_{p}(0,1)$; this basis is unconditional for $p=2$. Moreover, the conditions of the uniform convergence of spectral expansions of continuous functions in the system of eigenfunctions of the problems (1.4) and (1.5) were established, respectively in $[12,21]$.

Note that the problem (1.5) is a special case of the problem (1.1)-(1.3) under the condition $b=0$ while the problem (1.4) is a special case of the problem (1.5) under the conditions $q(x) \equiv 0$ and $a>0$.

\section{Existence of eigenvalues and asymptotic formulae for eigenvalues and eigenfunctions of the problem (1.1)-(1.3)}

In this section, we prove existence of eigenvalues of the problem (1.1)-(1.3) and then we give asymptotic formulae for eigenvalues and eigenfunctions of the same problem.

Let $\psi(x, \lambda)$ denote the solution of the equation (1.1) which satisfies the initial conditions

$$
\psi(0, \lambda)=0, \psi^{\prime}(0, \lambda)=1 .
$$

The eigenvalues of the problem (1.1)-(1.3) are the zeros of the entire function

$$
F(\lambda)=1-\lambda\left(a \psi(1, \lambda)+b \psi^{\prime}(1, \lambda)\right)
$$

or the roots of the equation

$$
1-\lambda\left(a \psi(1, \lambda)+b \psi^{\prime}(1, \lambda)\right)=0 .
$$

This function does not vanish because $F(0)=1$.

Let $E$ be the set of the roots of the equation

$$
a\left(\psi(1, \lambda)+\lambda \frac{\partial \psi(1, \lambda)}{\partial \lambda}\right)+b\left(\psi^{\prime}(1, \lambda)+\lambda \frac{\partial \psi^{\prime}(1, \lambda)}{\partial \lambda}\right)=0,
$$

where $a$ and $b$ are fixed numbers. $E$ is a countable set. Henceforth, we assume that $F(\lambda) \neq 0$ for all $\lambda \in E$.

Theorem 2.1. All eigenvalues of the boundary value problem (1.1)-(1.3) are simple and they form an infinite sequence $\lambda_{n}(n=0,1,2, \ldots)$ which has no finite limit point. Moreover, the asymptotic formulae

$$
\lambda_{n}=\left[\left(n-\frac{1}{2}\right) \pi\right]^{2}+O(1)
$$




$$
\psi_{n}(x)=\psi\left(x, \lambda_{n}\right)=\frac{\sin \left(n-\frac{1}{2}\right) \pi x}{n \pi}+O\left(n^{-2}\right)
$$

are valid, where $\psi_{n}(x)$ is the eigenfunction corresponding to $\lambda_{n}$ for sufficiently large numbers of $n$.

Proof. Let $\lambda=s^{2}$ and $s=\sigma+i t$. Then,

$$
\psi(x, \lambda)=\frac{\sin s x}{s}+\frac{1}{s} \int_{0}^{x} q(\tau) \psi(\tau, \lambda) \sin s(x-\tau) d \tau
$$

and there exists $s_{0}>0$ such that for $|s|>s_{0}$ the estimate

$$
\psi(x, \lambda)=\frac{\sin s x}{s}+O\left(e^{|t| x}|s|^{-2}\right)
$$

is valid [18, Chapter I, Lemma 1.2.1 and Lemma 1.2.2], where the function $O\left(e^{|t| x}|s|^{-2}\right)$ is an entire function of $s$ for any fixed $x$ in [0,1]. Moreover, (2.6) holds uniformly in $x$ for $0 \leq x \leq 1$. Therefore, by differentiating (2.5) with respect to $x$, we have

$$
\psi^{\prime}(x, \lambda)=\cos s x+\int_{0}^{x} q(\tau) \psi(\tau, \lambda) \cos s(x-\tau) d \tau .
$$

From here and (2.6), we obtain

$$
\psi^{\prime}(x, \lambda)=\cos s x+O\left(e^{|t| x}|s|^{-1}\right) .
$$

Thus, according to (2.6) and (2.8), the equation (2.2) takes the form

$$
s^{2} \cos s+O\left(e^{|t|}|s|\right)=0 \text {. }
$$

The inequality

$$
\left|s^{2} \cos s\right| \geq \frac{1}{4} e^{|t|}|t|^{2}
$$

is valid for sufficiently large $|t|$. From here, we obtain that the limit of modulus of the first summand of the equation (2.9) is $+\infty$ as $|t| \rightarrow \infty$. So, there exists $M>0$ such that $|t| \leq M$ for any solution $s$ of equation (2.9). Then, the equation (2.9) is equivalent to the equation

$$
s^{2} \cos s+O(|s|)=0 .
$$

It is taken into account that $s=0$ is not a root of equation (2.10) since $\lambda=0$ is not an eigenvalue of the boundary value problem (1.1)-(1.3). It is obvious that the roots of the equation (2.10) are simple. Otherwise, $\lambda$ is a multiple root of the equation (2.2) and this is contrary to $F(\lambda) \neq 0(\lambda \in E)$.

We choose a positive number $H$ such that all the roots of the equation (2.10) lie in the domain $\{z \in \mathbb{C}:|\operatorname{Im} z| \leq H\}$ and the condition $\sinh H \geq 1$ holds. We now find the number of the roots of equation (2.10) inside

$$
D_{n, 1}=\{z \in \mathbb{C}:|\operatorname{Im} z| \leq H,|\operatorname{Re} z| \leq n \pi\},
$$

for sufficiently large $n$.

It is well known that the inequalities

$$
|\cos z| \geq|\cos x|,|\cos z| \geq|\sinh y|
$$

are valid, where $z=x+i y \in \mathbb{C}$. From (2.11), if $z=x \mp i H,-n \pi \leq x \leq n \pi$, then $|\cos z| \geq \sinh H \geq 1$ and if $z=\mp n \pi+i y,-H \leq y \leq H$, then $|\cos z| \geq|\cos n \pi|=1$. By virtue of Rouche's theorem [4, Chapter IV, Theorem 6.2], for sufficiently large $n$, there are as many zeros of equation (2.10) inside the domain $D_{n, 1}$ as of the equation $s^{2} \cos s=0$, i.e., $2 n+2$. 
Since $\lambda=s^{2}$, we only need to consider the roots of the equation (2.10) which satisfy the condition $s \in D=\left\{z \in \mathbb{C}:-\frac{\pi}{2}<\arg z \leq \frac{\pi}{2}\right\}$ for the eigenvalues of the boundary value problem (1.1)-(1.3). It is obvious that the number of the roots of the equation (2.10) are $n+1$ inside the domain

$$
D_{n, 2}=\left\{z \in \mathbb{C}:-\frac{\pi}{2}<\arg z \leq \frac{\pi}{2}, \operatorname{Re} z \leq n \pi\right\} .
$$

By using Rouche's theorem again, it is easy to see that there is only one root of equation $(2.10)$ at the neighborhood $O\left(n^{-1}\right)$ of the number $\left(n-\frac{1}{2}\right) \pi(n \in \mathbb{N})$ for sufficiently large $n$.

We number the roots (which satisfy the condition $s \in D$ ) of the equation (2.10) in ascending order of $\operatorname{Re} s_{n}(n=0,1, \ldots)$. By virtue of these discussions, we obtain

$$
s_{n}=\left(n-\frac{1}{2}\right) \pi+O\left(n^{-1}\right) .
$$

The formulae (2.3) and (2.4) are established by the equalities $\lambda_{n}=s_{n}^{2},(2.6)$, and (2.12). The proof of theorem 2.1 is completed.

\section{The basis property of the system of eigenfunctions of the problem (1.1)-(1.3) in $L_{p}(0,1)$}

In this section, we give the minimality of the system of eigenfunctions of the problem (1.1)-(1.3) with one function deleted and prove the basicity in $L_{p}(0,1)$ of this system.

Let $q(x)=q(1-x)(0 \leq x \leq 1)$ and $\psi_{k}(1)=0$. The function $\xi_{k}(x)=\psi_{k}(1-x)+$ $b \lambda_{k} \psi_{k}(x)$ is a solution of the equation (1.1) for $\lambda=\lambda_{k}$. By (1.3) and (2.1), we have $\xi_{k}(1)=\xi_{k}^{\prime}(1)=0$. Consequently, $\xi_{k}(x)=0,0 \leq x \leq 1$. In other words,

$$
\psi_{k}(1-x)=-b \lambda_{k} \psi_{k}(x), 0 \leq x \leq 1 .
$$

From the last equality, we obtain $\left(b \lambda_{k}\right)^{2}=1$. Hence, since $b^{2} \neq \frac{1}{\lambda_{n}^{2}}(n=0,1, \ldots)$, then $\psi_{n}(1) \neq 0$.

Firstly, let us give some lemmas to be used in the proof of our theorems.

Lemma 3.1. The equalities

$$
\begin{gathered}
\frac{\partial \psi\left(1, \lambda_{n}\right)}{\partial \lambda}=O\left(n^{-3}\right) \\
\frac{\partial \psi^{\prime}\left(1, \lambda_{n}\right)}{\partial \lambda}=\frac{(-1)^{n}}{2 n \pi}+O\left(n^{-2}\right)
\end{gathered}
$$

hold for sufficiently large $n$.

Proof. By using (2.5) and (2.7), we obtain the equalities

$$
\begin{aligned}
\frac{\partial \psi(1, \lambda)}{\partial s} & =\frac{\cos s}{s}-\frac{\sin s}{s^{2}}-\frac{1}{s^{2}} \int_{0}^{1} q(\tau) \psi(\tau, \lambda) \sin s(1-\tau) d \tau+ \\
& +\frac{1}{s} \int_{0}^{1}(1-\tau) q(\tau) \psi(\tau, \lambda) \cos s(1-\tau) d \tau \\
& +\frac{1}{s} \int_{0}^{1} q(\tau) \frac{\partial \psi(\tau, \lambda)}{\partial s} \sin s(1-\tau) d \tau,
\end{aligned}
$$




$$
\begin{aligned}
\frac{\partial \psi^{\prime}(1, \lambda)}{\partial s} & =-\sin s+\int_{0}^{1} q(\tau) \frac{\partial \psi(\tau, \lambda)}{\partial s} \cos s(1-\tau) d \tau \\
& -\int_{0}^{1}(1-\tau) q(\tau) \psi(\tau, \lambda) \sin s(1-\tau) d \tau .
\end{aligned}
$$

Using the equalities (2.4) and (2.12), it is not hard to see the estimates

$$
\begin{gathered}
\frac{\partial \psi\left(1, \lambda_{n}\right)}{\partial s}=\frac{1}{s_{n}} \int_{0}^{1} q(\tau) \frac{\partial \psi\left(\tau, \lambda_{n}\right)}{\partial s} \sin s_{n}(1-\tau) d \tau+O\left(n^{-2}\right), \\
\frac{\partial \psi^{\prime}\left(1, \lambda_{n}\right)}{\partial s}=(-1)^{n}+\int_{0}^{1} q(\tau) \frac{\partial \psi\left(\tau, \lambda_{n}\right)}{\partial s} \cos s_{n}(1-\tau) d \tau+O\left(n^{-1}\right) .
\end{gathered}
$$

Let $M_{n}=\max _{0 \leq x \leq 1}\left|\frac{\partial \psi\left(x, \lambda_{n}\right)}{\partial s}\right|$. By virtue of (3.3), the inequality

$$
M_{n} \leq C_{1}\left(\frac{M_{n}}{\left|s_{n}\right|}+\frac{1}{n^{2}}\right)
$$

holds, where $C_{1}$ is a constant which is independent of $n$. From last inequality and (2.12), the estimate

$$
M_{n} \leq \frac{C_{2}}{n^{2}}
$$

is valid for sufficiently large $n$, where $C_{2}$ is a constant which is independent of $n$. Thus, estimate (3.1) is obtained by (3.3) and (3.5). By using (3.4) and (3.5), we obtain

$$
\frac{\partial \psi^{\prime}\left(1, \lambda_{n}\right)}{\partial s}=(-1)^{n}+O\left(n^{-1}\right)
$$

From here, estimate (3.2) is obtained directly. The proof of the lemma 3.1 is completed.

Henceforth, we will assume that the relation $q(x)=q(1-x)(0 \leq x \leq 1)$ is valid in lemma and theorems established for the investigation of spectral properties of the problem (1.1)-(1.3) in this study.

Lemma 3.2. The following equalities are satisfied:

$$
\begin{gathered}
\left(\psi_{n}(x), \overline{\psi_{m}(1-x)}\right)=\frac{\psi_{n}(1)-\psi_{m}(1)}{\lambda_{m}-\lambda_{n}}, \quad(n \neq m ; n, m=0,1,2, \ldots) \\
\left(\psi_{n}(x), \overline{\psi_{n}(1-x)}\right)=-\frac{\partial \psi\left(1, \lambda_{n}\right)}{\partial \lambda}, \quad(n=0,1,2, \ldots) \\
\left(\psi_{n}(x), \overline{\psi_{m}(x)}\right)=\frac{\lambda_{m} \psi_{m}(1)-\lambda_{n} \psi_{n}(1)}{b \lambda_{n} \lambda_{m}\left(\lambda_{m}-\lambda_{n}\right)}, \quad(n \neq m ; n, m=0,1,2, \ldots) \\
\left(\psi_{n}(x), \overline{\psi_{n}(x)}\right)=\psi_{n}^{\prime}(1) \frac{\partial \psi\left(1, \lambda_{n}\right)}{\partial \lambda}-\psi_{n}(1) \frac{\partial \psi^{\prime}\left(1, \lambda_{n}\right)}{\partial \lambda},(n=0,1,2, \ldots)
\end{gathered}
$$

where $(f, g)=\int_{0}^{1} f(x) \overline{g(x)} d x$.

Proof. It is easily seen that from equation (1.1), the equality

$$
\frac{d}{d x}\left\{\psi_{n}^{\prime}(x) \psi_{m}(1-x)+\psi_{n}(x) \psi_{m}^{\prime}(1-x)\right\}=\left(\lambda_{m}-\lambda_{n}\right) \psi_{n}(x) \psi_{m}(1-x)
$$

holds for $0 \leq x \leq 1$. Integrating with respect to $x$ from 0 to 1 , we obtain

$$
\left(\lambda_{m}-\lambda_{n}\right)\left(\psi_{n}(x), \overline{\psi_{m}(1-x)}\right)=\left.\left(\psi_{n}^{\prime}(x) \psi_{m}(1-x)+\psi_{n}(x) \psi_{m}^{\prime}(1-x)\right)\right|_{0} ^{1} .
$$

The equality (3.6) is obtained by the last equation and the initial conditions (2.1). 
Similarly, we obtain the equality

$$
\frac{d}{d x}\left\{\psi_{n}^{\prime}(x) \psi(1-x, \lambda)+\psi_{n}(x) \psi^{\prime}(1-x, \lambda)\right\}=\left(\lambda-\lambda_{n}\right) \psi_{n}(x) \psi(1-x, \lambda)
$$

for $\lambda \neq \lambda_{n}$. From here and (2.1), the equality

$$
\left(\psi_{n}(x), \overline{\psi(1-x, \lambda)}\right)=\frac{\psi_{n}(1)-\psi(1, \lambda)}{\lambda-\lambda_{n}}
$$

is valid. Taking the limit as $\lambda \rightarrow \lambda_{n}$ in the last equality, we obtain (3.7). Equalities (3.8) and (3.9) are verified similarly.

Firstly, let us verify that the system $\psi_{n}(x)(n=0,1, \ldots ; n \neq r)$ is minimal in the space $L_{2}(0,1)$ by the following theorem. It suffices to prove the existence of the system $\varphi_{n}(x)$ $(n=0,1, \ldots ; n \neq r)$ which is biorthogonally conjugate to the system $\psi_{n}(x)(n=0,1, \ldots ; n \neq r)$ in the space $L_{2}(0,1)$ (see [24, Chapter I, §6, Theorem 6.1]).

Theorem 3.3. If $b^{2} \neq \frac{1}{\lambda_{n}^{2}}(n=0,1, \ldots)$, then the system $\psi_{n}(x)(n=0,1, \ldots ; n \neq r)$, where $r$ is an arbitrary fixed non-negative integer, is minimal in $L_{2}(0,1)$.

Proof. Since $\lambda_{n}$ is a simple root of the equation (2.2) for every $n$, we have

$$
a\left(\psi_{n}(1)+\lambda_{n} \frac{\partial \psi\left(1, \lambda_{n}\right)}{\partial \lambda}\right)+b\left(\psi_{n}^{\prime}(1)+\lambda_{n} \frac{\partial \psi^{\prime}\left(1, \lambda_{n}\right)}{\partial \lambda}\right) \neq 0
$$

or

$$
\frac{1}{\lambda_{n}}+\lambda_{n}\left(a \frac{\partial \psi\left(1, \lambda_{n}\right)}{\partial \lambda}+b \frac{\partial \psi^{\prime}\left(1, \lambda_{n}\right)}{\partial \lambda}\right) \neq 0
$$

The functions $\varphi_{n}(x)(n=0,1, \ldots ; n \neq r)$ are defined by the following:

$$
\overline{\varphi_{n}(x)}=a_{n}\left[\frac{\psi_{n}(1-x)+a \lambda_{n} \psi_{n}(x)}{\psi_{n}(1)}-\frac{\psi_{r}(1-x)+a \lambda_{r} \psi_{r}(x)}{\psi_{r}(1)}\right],
$$

where $a_{n}=-\left(\frac{1}{\lambda_{n}}+\lambda_{n}\left(a \frac{\partial \psi\left(1, \lambda_{n}\right)}{\partial \lambda}+b \frac{\partial \psi^{\prime}\left(1, \lambda_{n}\right)}{\partial \lambda}\right)\right)^{-1}$. Because of the assumption $\left(b^{2} \neq \frac{1}{\lambda_{n}^{2}}\right)$ of the theorem, $\psi_{n}(1) \neq 0$ for all $n$.

Assume that $n \neq m, r ; m \neq r$. The equality

$$
\begin{aligned}
\left(\psi_{n}, \varphi_{m}\right) & =a_{n}\left[\frac{\left(\psi_{n}(x), \overline{\psi_{m}(1-x)}\right)+b \lambda_{m}\left(\psi_{n}(x), \overline{\psi_{m}(x)}\right)}{\psi_{m}(1)}-\frac{\left(\psi_{n}(x), \overline{\psi_{r}(1-x)}\right)+b \lambda_{r}\left(\psi_{n}(x), \overline{\psi_{r}(x)}\right)}{\psi_{r}(1)}\right] \\
& =a_{n}\left[\frac{\frac{\psi_{n}(1)-\psi_{m}(1)}{\lambda_{m}-\lambda_{n}}+b \lambda_{m} \frac{\lambda_{m} \psi_{m}(1)-\lambda_{n} \psi_{n}(1)}{b \lambda_{n} \lambda_{m}\left(\lambda_{m}-\lambda_{n}\right)}}{\psi_{m}(1)}-\frac{\frac{\psi_{n}(1)-\psi_{r}(1)}{\lambda_{m}-\lambda_{r}}+b \lambda_{r} \frac{\lambda_{r} \psi_{r}(1)-\lambda_{n} \psi_{n}(1)}{b \lambda_{n} \lambda_{r}\left(\lambda_{r}-\lambda_{n}\right)}}{\psi_{r}(1)}\right] \\
& =a_{n}\left(\frac{1}{\lambda_{n}}-\frac{1}{\lambda_{n}}\right)=0
\end{aligned}
$$

holds by (3.6) and (3.8).

Assume that $n \neq r$. The equality

$$
\begin{aligned}
\left(\psi_{n}, \varphi_{n}\right) & =a_{n}\left[\frac{\left(\psi_{n}(x) \overline{\psi_{n}(1-x)}\right)+b \lambda_{n}\left(\psi_{n}(x), \overline{\psi_{n}(x)}\right)}{\psi_{n}(1)}-\frac{\left(\psi_{n}(x), \overline{\psi_{r}(1-x)}\right)+b \lambda_{r}\left(\psi_{n}(x), \overline{\psi_{r}(x)}\right)}{\psi_{r}(1)}\right] \\
& =a_{n}\left[\frac{-\frac{\partial \psi\left(1, \lambda_{n}\right)}{\partial \lambda}+b \lambda_{n}\left(\psi_{n}^{\prime}(1) \frac{\partial \psi\left(1, \lambda_{n}\right)}{\partial \lambda}-\psi_{n}(1) \frac{\partial \psi^{\prime}\left(1, \lambda_{n}\right)}{\partial \lambda}\right)}{\psi_{n}(1)}-\frac{1}{\lambda_{n}}\right] \\
& =a_{n}\left(-a \lambda_{n} \frac{\partial \psi\left(1, \lambda_{n}\right)}{\partial \lambda}+b \lambda_{n} \frac{\partial \psi^{\prime}\left(1, \lambda_{n}\right)}{\partial \lambda}-\frac{1}{\lambda_{n}}\right)=1
\end{aligned}
$$

is valid by (3.6)-(3.9). 
Theorem 3.4. If $b^{2} \neq \frac{1}{\lambda_{n}^{2}}(n=0,1, \ldots)$, then the system $\psi_{n}(x)(n=0,1, \ldots ; n \neq r)$, where $r$ is an arbitrary fixed non-negative integer, is a basis in the space $L_{p}(0,1)$ and this basis is unconditional in the space $L_{2}(0,1)$.

Proof. We need the asymptotic formulae of $\varphi_{n}(x)(n=0,1, \ldots ; n \neq r)$ to prove the basicity of the system $\psi_{n}(x)(n=0,1, \ldots ; n \neq r)$ in the space $L_{p}(0,1)$.

Using (2.3), (3.1), and (3.2), the estimate

$$
a_{n}=\frac{2(-1)^{n-1}}{b n \pi}+O\left(n^{-2}\right)
$$

holds for sufficiently large $n$. From here and (2.4), it is not hard to see the asymptotic formulae

$$
\overline{\varphi_{n}(x)}=2 n \pi \sin \left(n-\frac{1}{2}\right) \pi x+O(1) .
$$

The systems $y_{n}(x)(n=0,1, \ldots)$ and $u_{n}(x)(n=0,1, \ldots)$ are defined by the following:

$$
\begin{aligned}
& y_{n}(x)=s_{n} \sqrt{2} \psi_{n}(x), \\
& u_{n}(x)=\frac{1}{\overline{s_{n}} \sqrt{2}} \varphi_{n}(x) .
\end{aligned}
$$

We can easily verify that

$$
\left(y_{n}, u_{m}\right)=\delta_{n m}, \quad(n, m=0,1, \ldots ; n, m \neq r),
$$

where $\delta_{n m}$ is the Kronecker symbol. Hence, the system $u_{n}(x)(n=0,1, \ldots ; n \neq r)$ is biorthogonally conjugate to the system $y_{n}(x)(n=0,1, \ldots ; n \neq r)$.

By virtue of (2.4) and (3.11), the estimates

$$
\begin{aligned}
& y_{n}(x)=\sqrt{2} \sin \left(n-\frac{1}{2}\right) \pi x+O\left(n^{-1}\right), \\
& u_{n}(x)=\sqrt{2} \sin \left(n-\frac{1}{2}\right) \pi x+O\left(n^{-1}\right)
\end{aligned}
$$

holds for sufficiently large $n$.

We denote the system $e_{n}(x)(n=1,2, \ldots)$ by the following:

$$
e_{n}(x)=\sqrt{2} \sin \left(n-\frac{1}{2}\right) \pi x
$$

Note that the system $e_{n}(x)(n=1,2, \ldots)$ is a basis of the space $L_{p}(0,1)$ and this is orthonormal for $p=2$ [3, Chapter VIII, §20, Theorem 2].

Let us compare the system $y_{n}(x)(n=0,1, \ldots ; n \neq r)$ with the system $e_{n}(x)(n=1,2, \ldots)$. By 3.14, the following inequality is valid for sufficiently large $n$ :

$$
\left\|y_{n}(x)-\sqrt{2} \sin \left(n-\frac{1}{2}\right) \pi x\right\| \leq \frac{C_{3}}{n},
$$

where $C_{3}$ is independent of $n$. From this inequality, we obtain that the series

$$
\sum_{n=1}^{r}\left\|y_{n-1}(x)-e_{n}(x)\right\|^{2}+\sum_{n=r+1}^{\infty}\left\|y_{n}(x)-e_{n}(x)\right\|^{2}
$$

is convergent (for $r=0$, the first sum is absent). Thus, the system $y_{n}(x)(n=0,1, \ldots ; n \neq r)$ is quadratically close to the system $e_{n}(x)(n=1,2, \ldots)$.

Since the system $y_{n}(x)(n=0,1, \ldots ; n \neq r)$ is minimal in the space $L_{2}(0,1)$, it is a Riesz basis in this space [5, Chapter VI, §2.4, Theorem 2.3].

The first part of Theorem 3.4 is proven. 
Note that the system $e_{n}(x)(n=1,2, \ldots)$ is a basis in $L_{p}(0,1)$, then there exists a constant $M_{p}>0$ ensuring the inequality

$$
\left\|\sum_{n=1}^{N}\left(f, e_{n}\right) e_{n}\right\|_{p} \leq M_{p}\|f\|_{p}, \quad N=1,2, \ldots
$$

for any function $f \in L_{p}(0,1)$, where $\|\cdot\|_{p}$ means the norm in $L_{p}(0,1)$ [9, Chapter I, $\S 4$, Theorem 6].

Let $1<p<2$ and $p$ be fixed. Since the system $y_{n}(x)(n=0,1, \ldots ; n \neq r)$ is complete in $L_{2}(0,1)$, then this system is complete in $L_{p}(0,1)$ as well. Consequently [9, Chapter VIII, $\S 4$, Theorem 6], in order to prove the basicity of this system in $L_{p}(0,1)$, it is enough to prove the existence of a constant $M>0$ ensuring the inequality

$$
\left\|\sum_{n=0, n \neq r}^{N}\left(f, u_{n}\right) y_{n}\right\|_{p} \leq M\|f\|_{p}, \quad N=1,2, \ldots
$$

for any function $f \in L_{p}(0,1)$.

Note that there exists $\widetilde{M_{1}}>0$ such that the inequality

$$
\left\|\left(f, u_{0}\right) y_{0}\right\|_{p} \leq \widetilde{M_{1}}\|f\|_{p}
$$

holds for every $f \in L_{p}(0,1)$. So, the inequality (3.17) is equivalent to the inequality

$$
E_{N}(f)=\left\|\sum_{n=1, n \neq r}^{N}\left(f, u_{n}\right) y_{n}\right\|_{p} \leq \widetilde{M}\|f\|_{p}, \quad N=1,2, \ldots
$$

where $\widetilde{M}$ is positive constant. According to (3.14), (3.15), and (3.18), the inequality

$$
E_{N}(f) \leq E_{N, 1}(f)+E_{N, 2}(f)+E_{N, 3}(f)+E_{N, 4}(f)
$$

is valid, where $N=1,2, \ldots$ and

$$
\begin{gathered}
E_{N, 1}(f)=\left\|\sum_{n=1, n \neq r}^{N}\left(f, e_{n}\right) e_{n}\right\|_{p}, \quad E_{N, 2}(f)=\left\|\sum_{n=1, n \neq r}^{N}\left(f, e_{n}\right) O\left(n^{-1}\right)\right\|_{p} \\
E_{N, 3}(f)=\left\|\sum_{n=1, n \neq r}^{N}\left(f, O\left(n^{-1}\right)\right) e_{n}\right\|_{p}, \quad E_{N, 4}(f)=\left\|\sum_{n=1, n \neq r}^{N}\left(f, O\left(n^{-1}\right)\right) O\left(n^{-1}\right)\right\|_{p} .
\end{gathered}
$$

By virtue of (3.16), the inequality

$$
E_{N, 1}(f) \leq \text { const. }\|f\|_{p}
$$

holds. From the Riesz theorem [26, Chapter XII, §2, Theorem 2.8] it follows that

$$
\begin{aligned}
E_{N, 2}(f) & \leq \text { const. } \sum_{n=1}^{N}\left|\left(f, e_{n}\right)\right| n^{-1} \leq \\
& \leq \text { const. }\left(\sum_{n=1}^{N}\left|\left(f, e_{n}\right)\right|^{q}\right)^{\frac{1}{q}}\left(\sum_{n=1}^{N} n^{-p}\right)^{\frac{1}{p}} \leq \text { const. }\|f\|_{p},
\end{aligned}
$$

where $\frac{1}{p}+\frac{1}{q}=1$. Further,

$$
\begin{aligned}
E_{N, 3}(f) & \leq\left\|\sum_{n=1}^{N}\left(f, O\left(n^{-1}\right)\right) e_{n}\right\|_{2_{1}}=\left(\sum_{n=1}^{N}\left|\left(f, O\left(n^{-1}\right)\right)\right|^{2}\right)^{\frac{1}{2}} \leq \\
& \leq \text { const. }\|f\|_{1}\left(\sum_{n=1}^{N} n^{-2}\right)^{\frac{1}{2}} \leq \text { const. }\|f\|_{p} .
\end{aligned}
$$


Moreover,

$$
E_{N, 4}(f) \leq \text { const. }\|f\|_{1} \sum_{n=1}^{N} n^{-2} \leq \text { const. }\|f\|_{p} .
$$

The inequality (3.18) is a consequence of the inequalities (3.19)-(3.23). Thus, the basicity of the system $y_{n}(x)(n=0,1, \ldots ; n \neq r)$ in the space $L_{p}(0,1)$ for $1<p<2$ is proven.

Let $2<p<\infty$ and $\frac{1}{p}+\frac{1}{q}=1$. It is evident that the system $u_{n}(x)(n=0,1, \ldots ; n \neq r)$ is a basis in the space $L_{p}(0,1)$. Consequently, this system is complete in the space $L_{q}(0,1)$. Note that $1<p<2$. By means of absolute analogous discussions used above, the basicity in $L_{q}(0,1)$ of the system $u_{n}(x)(n=0,1, \ldots ; n \neq r)$ is proven. Hence, the basicity in $L_{p}(0,1)(2<p<\infty)$ of the system $y_{n}(x)(n=0,1, \ldots ; n \neq r)$ follows. The proof of Theorem 3.4 is completed.

\section{References}

[1] Y.N. Aliyev, On the basis properties of Sturm-Liouville problems with decreasing affine boundary conditions, Proc. IMM of NAS, 24, 35-52, 2006.

[2] Y.N. Aliyev and N.B. Kerimov, The basis property of Sturm-Liouville problems with boundary conditions depending quadratically on the eigenparameter, Arab. J. Sci. Eng. 33 (1A), 123-136, 2008.

[3] N.K. Bary, Treatise on Trigonometric Series, Vol II., Macmillian, New York, 1964.

[4] M.A. Evgrafov, Analytic Function (in Russian), Nauka, Moskow, 1965; trans. W.B. Saunders Comp., Philadephia and London, 1966.

[5] I.C. Gohberg and M.G. Krein, Introduction to the Theory of Linear Nonselfadjoint Operators, Moscow, 1965; Trans. Math. Monogr., Amer. Math. Soc., Rhode Island, 18, 1969.

[6] S. Goktas, N.B. Kerimov, and E.A. Maris, On the uniform convergence of spectral expansions for a spectral problem with a boundary condition rationally depending on the eigenparameter, J. Korean Math. Soc. 54 (4), 1175-1187, 2017.

[7] T. Gulsen, E. Yilmaz, and H. Koyunbakan, An inverse nodal problem for differential pencils with complex spectral parameter dependent boundary conditions, New Trends Math. Sci. 5 (1), 137-144, 2017.

[8] N.Yu. Kapustin and E.I. Moiseev, The basis property in of the systems of eigenfunctions corresponding to two problems with a spectral parameter in the boundary conditions, Diff. Eq. 36 (10), 1498-1501, 2000.

[9] B.S. Kashin and A.A. Saakyan, Orthogonal Series, Trans. Math. Monogr., Amer. Math. Soc. Providence, 75, 1989.

[10] N.B. Kerimov and Y.N. Aliyev, The basis property in $L_{p}(0,1)$ of the boundary value problem rationally dependent on the eigenparameter, Studia Math. 174 (2), 201-212, 2006.

[11] N.B. Kerimov and Kh.R. Mamedov, On one boundary value problem with a spectral parameter in the boundary conditions, Siberian Math. J. 40 (2), 325-335, 1999.

[12] N.B. Kerimov and E.A. Maris, On the basis properties and convergence of expansions in terms of eigenfunctions for a spectral problem with a spectral parameter in the boundary condition, Proc. IMM of NAS (Special Issue) 40, 245-258, 2014.

[13] N.B. Kerimov and E.A. Maris, On the uniform convergence of the Fourier Series for one spectral problem with a spectral parameter in a boundary condition, Math. Methods Appl. Sci. 39 (9), 2298-2309, 2016.

[14] N.B. Kerimov and E.A. Maris, On the Uniform Convergence of Fourier Series Expansions for Sturm-Liouville Problems with a Spectral Parameter in the Boundary Conditions, Results Math. 73 (3), 102, 2018.

[15] N.B. Kerimov and V.S. Mirzoev, On the basis properties of one spectral problem with a spectral parameter in a boundary condition, Siberian Math. J. 44 (5), 813-816, 2003. 
[16] N.B. Kerimov and R.G. Poladov, Basis properties of the system of eigenfunctions in the Sturm- Liouville problem with a spectral parameter in the boundary conditions, Dokl. Math. 85 (1), 8-13, 2015.

[17] N.B. Kerimov, S. Goktas, and E.A. Maris, Uniform convergence of the spectral expansions in terms of root functions for a spectral problem, Electron. J. Differ. Equ. 80, 1-14, 2016.

[18] B.M. Levitan and I.S. Sargsjan, Sturm-Liouville and Dirac Operators, Kluwer Academic Publishers: Netherlands, 1991.

[19] Kh.R. Mamedov, On one boundary value problem with parameter in the boundary conditions, Spectr Theory Oper. Appl. 11, 117-121, 1997 (in Russian).

[20] Kh.R. Mamedov, On a basic problem for a second order differential equation with a discontinuous coefficient and a spectral parameter in the boundary conditions, Proc. Seventh Internat. Conf. Geometry, Integrability and Quantization, Institute of Biophysics and Biomedical Engineering Bulgarian Academy of Sciences, 218-225, 2006.

[21] D.B. Marchenkov, On the convergence of spectral expansions of functions for problems with a spectral parameter in a boundary condition, Diff. Eq. 41, 1496-1500, 2005.

[22] D.B. Marchenkov, Basis property in $L_{p}(0,1)$ of the system of eigenfunctions corresponding to a problem with a spectral parameter in the boundary condition, Diff. Eq. 42 (6), 905-908, 2006.

[23] A. Neamaty and Sh. Akbarpoor, Numerical solution of inverse nodal problem with an eigenvalue in the boundary condition, Inverse Probl. Sci. Eng. 25 (7), 978-994, 2017.

[24] I. Singer, Bases in Banach Spaces I, Springer-Verlag Berlin Heidelberg, New York, 1970.

[25] E. Yilmaz and H. Koyunbakan, Reconstruction of potential function and its derivatives for Sturm-Liouville problem with eigenvalues in boundary condition, Inverse Prob. Sci. Eng. 18 (7), 935-944, 2010.

[26] A. Zygmund, Trigonometric Series, Vol. II, 2nd Ed., Cambridge University Press, New York, 1959. 